\author{
Methodik ÖR \\ Übungsklausur
}

Prof. Dr. Markus Ludwigs* und Wiss. Mit. Hannah Amann

\title{
Klausur im Kommunalrecht: Ausschluss aus dem Gemeinderat
}

https://doi.org/10.1515/jura-2017-0205

\section{SACHVERHALT}

$\mathrm{R}$ ist Mitglied des Gemeinderates der bayerischen Gemeinde D in Unterfranken. Er hatte im Jahr 2014 vor dem Verwaltungsgericht Würzburg - ebenso wie weitere 58 Bürger der Gemeinde - Klage gegen die Gebührenbescheide erhoben, mit denen die Wasser-, Abwasser- und Abfallgebühren für die Jahre 2012-2014 festgesetzt worden waren. Das Verwaltungsgericht gab einer dieser Klagen im Jahr 2015 statt und hob die betreffenden Bescheide auf, die dem Kläger gegenüber erlassen worden waren. Die übrigen Klagen, auch die von R, wurden dagegen bis zur rechtskräftigen Entscheidung über das »Musterverfahren« ausgesetzt.

Mit Schreiben vom 12. 01. 2016 lud der erste Bürgermeister die Gemeinderatsmitglieder, darunter auch den $\mathrm{R}$, form- und fristgerecht zur Gemeinderatssitzung am 19. 01. 2016. Zur Beratung und Entscheidung stand unter anderem die Frage, ob gegen das stattgebende Urteil des VG Würzburg Antrag auf Zulassung der Berufung gestellt werden soll. Außerdem sollte in der Sitzung über eine Änderungssatzung hinsichtlich der Wasser-, Abwasserund Abfallgebühren beraten und entschieden werden, um in Anbetracht der Entscheidung des VG Würzburg die Gebühren für die Kläger auch der ruhenden Verfahren rückwirkend neu festsetzen zu können. Dahinter stand der Plan, einen positiven Ausgang auch dieser Verfahren zu verhindern.

In der öffentlichen Sitzung des Gemeinderates vom 19. 01. 2016 wurde der Ausschluss von vier Gemeinderatsmitgliedern - einschließlich des $\mathrm{R}$ - von der Beratung und Entscheidung sowohl hinsichtlich des Zulassungsantrages

*Kontaktperson: Markus Ludwigs, der Autor ist Inhaber des Lehrstuhls für Öffentliches Recht und Europarecht an der Julius-Maximilians-Universität Würzburg

Hannah Amann, die Autorin ist Wiss. Mitarbeiterin und Doktorandin an diesem Lehrstuhl. gegen das Urteil des VG Würzburg als auch über den Erlass der Änderungssatzung mehrheitlich beschlossen. Die Betroffenen verließen daraufhin den Saal.

$\mathrm{R}$ ist der Ansicht, dass der Ausschluss rechtswidrig war. Er habe die Sitzung in keiner Weise gestört und sehe nicht, inwiefern die vorgenommenen Entscheidungen ihn hätten begünstigen sollen. Bei der Entscheidung über den Berufungszulassungsantrag gehe es schließlich nicht um sein eigenes Verfahren, sondern um das eine, welches das VG Würzburg vorab als "Musterverfahren« im Sinne von $\S 93$ a VwGO durchgeführt habe. Sein Gebührenrechtsstreit sei davon völlig unabhängig.

Auch hinsichtlich der Satzung versteht R nicht, weshalb er von einer Mitentscheidung ausgeschlossen wurde. Eine Satzung, welche von der Gemeinde auf Grund ihres Selbstbestimmungsrechts erlassen werden dürfe, könne ihn als abstrakt-generelle Regelung doch gar nicht individuell betreffen. So würde er als Gemeindevertreter bei der Ausübung seines Mandats viel zu sehr eingeschränkt.

Der Gemeinderat ist der Meinung, dass der Ausgang des »Musterverfahrens « für die Klage des R sehr wohl von Relevanz sei. Ein solches werde doch gerade dann durchgeführt, wenn zu erwarten sei, dass dies im Rahmen der Prozessökonomie der Beschleunigung und Vereinfachung der ruhenden Verfahren diene. Hinsichtlich des Beschlusses der Änderungssatzung sei zu berücksichtigen, dass es um rückwirkende Änderungen gehe, die gerade die Kläger beträfen.

Bearbeitervermerk: Prüfen Sie, ob eine verwaltungsgerichtliche Klage des $\mathrm{R}$ gegen den Sitzungsausschluss Aussicht auf Erfolg hat. Dabei ist auf alle aufgeworfenen Rechtsfragen, gegebenenfalls hilfsgutachterlich, einzugehen. Von der Zulässigkeit des Erlasses einer rückwirkenden Änderungssatzung ist auszugehen.

\section{LÖSUNGSVORSCHLAG}

Ein verwaltungsgerichtliches Vorgehen gegen den Sitzungsausschluss des R hat Aussicht auf Erfolg, wenn die 
entsprechenden Sachentscheidungsvoraussetzungen für eine Klage vorliegen und soweit diese begründet ist. ${ }^{1}$

\section{A. Sachentscheidungs- voraussetzungen}

\section{Eröffnung des Verwaltungsrechtswegs}

Mangels Eingreifens einer aufdrängenden Sonderzuweisung, richtet sich die Eröffnung des Verwaltungsrechtswegs nach der Generalklausel des § 40 I 1 VwGO.

\section{1. Öffentlich-rechtliche Streitigkeit}

Vorliegend wird um den Ausschluss von der Gemeinderatssitzung gestritten. Es handelt sich um eine Streitigkeit zwischen einem Organteil (dem Ratsmitglied R) und einem Organ (dem Gemeinderat) derselben Gemeinde und daher um eine sogenannte Kommunalverfassungsstreitigkeit, in Form einer intraorganschaftlichen Streitigkeit. ${ }^{2}$ Eine Streitigkeit i.S.v. § 40 I 1 VwGO könnte deshalb zu verneinen sein, da somit eine intrapersonale Streitigkeit in Rede steht, bei der innerhalb eines Verwaltungsträgers gestritten wird, und keine interpersonale, bei der sich mehrere Rechtssubjekte gegenüberstehen. Fraglich ist, ob auch solche Innenrechtsstreitigkeiten von § 40 I 1 VwGO erfasst werden, da die VwGO grundsätzlich auf Außenrechtsstreitigkeiten zwischen einzelnen Rechtssubjekten zugeschnitten ist. $^{3}$

Nach der Ende des 19. und zu Beginn des 20. Jahrhunderts vertretenen Impermeabilitätstheorie (Grundsatz der Undurchdringlichkeit) wurden klagbare Rechte im Binnenbereich des Staates nicht anerkannt. ${ }^{4}$ Dieser Ansatz, wonach der Staat rechtlich undurchdringbar (impermeabel) sein sollte, ist jedoch überholt, da aus dem Rechtsstaatsprinzip (Art. 23 I 1, 20 III, 28 I 1 GG) folgt, dass auch Innenrechtsstreitigkeiten gerichtlich überprüfbar sein müssen.

1 Zum zwei- bzw. dreistufigen Prüfungsaufbau einer verwaltungsgerichtlichen Klage vgl. Hufen Verwaltungsprozessrecht, 10. Aufl., $\S 10$ Rn. 1 f; Fischer JURA 2003, 748.

2 Erbguth/Mann/Schubert Besonderes Verwaltungsrecht, 12. Aufl., Rn. 181; näher zur Unterscheidung zwischen Intra- und Inter-Organstreitigkeiten: Geis Kommunalrecht, 4. Aufl., § 25 Rn. 4; Ogorek JuS 2009, 511 (511).

3 Detterbeck Allgemeines Verwaltungsrecht, 14. Aufl., Rn. 1455.

4 Laband Das Staatsrecht des Deutschen Reiches, II, 5. Aufl.,

S. 181f.; s dazu auch Ogorek JuS 2009, 511 (512) m.w. N.
Da um einen Ausschluss wegen persönlicher Beteiligung gestritten wird, ist streitentscheidende Norm Art. 49 BayGO. Darin wird ein Hoheitsträger gerade einseitig berechtigt, weshalb die Norm nach der modifizierten Subjektstheorie ${ }^{5}$ öffentlich-rechtlicher Natur ist. Eine öffentlich-rechtliche Streitigkeit liegt somit vor.

\section{Nichtverfassungsrechtlicher Art}

Trotz der Bezeichnung »Kommunalverfassungsstreitigkeit« streiten vorliegend nicht Verfassungsorgane um Rechte und/oder Pflichten aus der Verfassung, sondern ein kommunales Organteil (Gemeinderatsmitglied) mit dem Organ (Gemeinderat) um Maßgaben aus der Gemeindeordnung. Eine doppelte Verfassungsunmittelbarkeit ${ }^{6}$ ist daher zu verneinen. Die Streitigkeit ist folglich nichtverfassungsrechtlicher Art.

\section{Keine abdrängende Sonderzuweisung}

Mangels Eingreifens einer abdrängenden Sonderzuweisung ist der Verwaltungsrechtsweg gem. § 40 I 1 VwGO eröffnet.

\section{Statthafte Klageart}

Die statthafte Klageart richtet sich nach dem klägerischen Begehren, § 88 VwGO. R begehrt die Klärung der Rechtswidrigkeit seines Ausschlusses als Gemeinderatsmitglied.

\section{Kommunalverfassungsstreitigkeit als Klage sui generis}

Unter Rekurs darauf, dass die Klagearten der VwGO auf Außenrechtsstreitigkeiten ausgerichtet seien, wird von einer Minderheitsansicht vorgebracht, der Kommunalverfassungsstreit sei als ein verwaltungsgerichtliches Klageverfahren eigener Art zu qualifizieren. ${ }^{7}$ Grundsätzlich ist eine

5 Hierzu statt vieler Maurer Allg. VerwR, 18. Aufl., § 3 Rn. 13.

6 Allgemein zur »doppelten Verfassungsunmittelbarkeit« z.B. Gärditz/Haack VwGO-Kommentar, 2013, § 40 Rn. 116 ff.; s. auch Bethge JuS 2001, 1100; im Kontext von Kommunalverfassungsstreitigkeiten: Becker/Heckmann/Kempen/Manssen/Becker Öffentliches Recht in Bayern, 6. Aufl., Teil 2 Rn. 273.

7 In diese Richtung Hufen Verwaltungsprozessrecht, 10. Aufl., § 21 Rn. 14; s. auch noch OVG Münster DVBl. 1973, 646 (647); a. A. die 
solche »Klage sui generis« zwar denkbar, da § 40 I 1 VwGO den Verwaltungsrechtsweg in »allen öffentlich-rechtlichen Streitigkeiten « eröffnet. ${ }^{8}$ Erforderlich ist sie aber nur, wenn das Begehren nicht bereits durch die bestehenden Klagearten abgedeckt wird. Ansonsten bestünde die Gefahr, dass besondere Sachurteilsvoraussetzungen der normierten Klagearten umgangen werden.

\section{Anfechtungsklage bzw. Fortsetzungsfeststellungsklage}

In Betracht kommt vorliegend zunächst eine Anfechtungsklage gem. § 42 I Alt. 1 VwGO. Dann müsste der Sitzungsausschluss ein Verwaltungsakt i.S.d. § $35 \mathrm{~S} .1$ VwVfG sein. Problematisch ist insoweit, ob das Merkmal der Außenwirkung erfüllt ist. Ordnungsmaßnahmen gegen ein Gemeinderatsmitglied entfalten nur dann Außenwirkung, wenn das Mitglied über seine Stellung als Organteil hinaus auch in der Stellung als Bürger betroffen ist. Da durch den Beschluss über den Ausschluss nur die organschaftlichen Rechte betroffen werden, handelt es sich mangels Außenwirkung nicht um einen Verwaltungsakt. ${ }^{9}$

Zwar wird von Teilen des Schrifttums vertreten, dass eine Begrenzung auf das Außenrechtsverhältnis nicht zwingend sei. Die Anerkennung von inter- und intraorganschaftlichen Streitigkeiten relativiere die Annahme, wonach das Erfordernis der Außenwirkung grundsätzlich dazu diene, den Innenbereich der Verwaltung von Rechtsstreitigkeiten frei zu halten. Daher könne Außenwirkung mit den organschaftlichen Rechtsbeziehungen gleichgesetzt werden, was zur Folge habe, dass eine Anfechtungsklage (bzw. eine Fortsetzungsfeststellungsklage) statthaft sei. ${ }^{10}$

Gegen diese Ansätze spricht aber zum einen, dass das Erfordernis der unmittelbaren Außenwirkung den Anwendungsbereich von Verwaltungsakten auf Rechtsbeziehungen zwischen selbstständigen Rechtsträgern beschränken

heute ganz hM; vgl. OVG Münster NVwZ-RR 2002, 135; Burgi Kommunalrecht, 5. Aufl., § 14 Rn. 10 f.; Ogorek JuS 2009, 511 (512); Erbguth/Mann/Schubert Besonderes Verwaltungsrecht, 12. Aufl., Rn. 182. 8 Hervorhebung der Verf.

9 Vgl. VGH Mannheim NVwZ-RR 1989, 91 (92); Becker/Heckmann/ Kempen/Manssen/Becker Öffentliches Recht in Bayern, 6. Aufl., Teil 2 Rn. 253; Erbguth/Mann/Schubert Besonderes Verwaltungsrecht, 12. Aufl., Rn. 182; Ogorek JuS 2009, 511 (512/513).

10 Hufen Verwaltungsprozessrecht, 10. Aufl., § 21 Rn. 10; Kopp/ Schenke/W.-R. Schenke VwGO, 22. Aufl., Vorb § 40 Rn. 7; Schenke Verwaltungsprozessrecht, 15. Aufl., § 5 Rn. 228; eine analoge Anwendung der Grundsätze der Anfechtungsklage befürwortet u.a. Lerche FS für Franz Knöpfle, 1996, S. 179 ff. soll. Diese bestehen innerhalb einer kommunalen Körperschaft gerade nicht. ${ }^{11}$ Im Übrigen wäre selbst bei Bejahung des Merkmals der Außenwirkung die Annahme eines Verwaltungsaktes schwerlich vertretbar. Zum einen lässt sich die Ausübung organschaftlicher Kompetenzen bei funktionaler Betrachtung nicht als Handeln einer Behörde qualifizieren. Zum anderen wird die Handlungsform des Verwaltungsaktes durch das hierin zum Ausdruck kommende Über-/Unterordnungsverhältnis gekennzeichnet. Ein solches besteht zwischen Organteilen jedoch nicht. ${ }^{12}$ Mangels Vorliegen eines Verwaltungsaktes ist daher auch eine Anfechtungsklage nach § 42 I Alt. 1 VwGO bzw. eine Fortsetzungsfeststellungsklage gem. § 113 I 4 VwGO nicht statthaft.

\section{Allgemeine Leistungsklage}

Des Weiteren könnte eine allgemeine Leistungsklage in Betracht kommen. Diese findet zwar keine explizite Verankerung in der VwGO, wird aber in mehreren Vorschriften (insb. §§ 43 II, 111 VwGO) implizit vorausgesetzt. Zum einen kommt eine herkömmliche Leistungsklage in Form der Vornahmeklage in Betracht, bei der die Verurteilung zu einer bestimmten Leistung erfolgt. Zum anderen hat der BayVGH der Leistungsklage bei Kommunalverfassungsstreitigkeiten auch kassatorische Wirkung verliehen, ${ }^{13}$ d.h., die Aufhebung der angegriffenen Maßnahme erfolgt durch das Gericht selbst. Für die allgemeine Leistungsklage mit einem solchen atypischen Aufhebungsantrag wird angeführt, dass auf diese Weise effektiver Rechtsschutz gewährt werde. ${ }^{14}$ Dem lässt sich indes entgegenhalten, dass der bestehende Rechtsschutz ausreichend ist und keiner Optimierung bedarf. Die Rechtsgestaltung ist der Leistungsklage wesensfremd. ${ }^{15}$ Zudem muss berücksichtigt werden, dass fehlerhafte Handlungen von Organen, insbesondere Beschlüsse des Gemeinderates in der Regel unwirksam sind, weshalb eine kassatorische Wirkung nicht erforderlich ist. ${ }^{16}$

11 Ogorek JuS 2009, 511 (513).

12 Ogorek JuS 2009, 511 (513).

13 BayVGH BayVBl. 1976, 753 (754); in Richtung einer systemwidrigen Feststellungsklage mit kassatorischer Wirkung dagegen: BayVGH BayVBl. 1988, 16 (16).

14 Becker/Heckmann/Kempen/Manssen/Becker Öffentliches Recht in Bayern, 6. Aufl., Teil 2 Rn. 276.

15 Ogorek JuS 2009, 511 (513).

16 Für eine Abkehr vom Nichtigkeitsdogma Schenke Verwaltungsprozessrecht, 15. Aufl., § 5 Rn. 228; Ehlers NVwZ 1990, 105 (107f.); wie hier Schoch JuS 1987, 783 (789); Becker/Heckmann/Kempen/Manssen/Becker Öffentliches Recht in Bayern, 6. Aufl., Teil 2 Rn. 276. 
Der Meinungsstreit ist indes nur dann relevant, wenn es nicht um erledigte Maßnahmen geht, also noch die Möglichkeit besteht, die Entscheidung zu revidieren und es dem Kläger darauf ankommt. Hier begehrt R aber der Sache nach die Feststellung der Rechtswidrigkeit seines durch Zeitablauf erledigten Ausschlusses. Die Leistungsklage passt daher nicht auf das Begehren des Klägers und ist unstatthaft. ${ }^{17}$

\section{Feststellungsklage}

Einschlägig könnte vielmehr eine Feststellungsklage gem. $\S 43 \mathrm{I}$ VwGO sein. Dann müsste es dem R um die Feststellung des Bestehens oder Nichtbestehens eines Rechtsverhältnisses gehen. Ein solches liegt vor, bei sich aus einem konkreten Sachverhalt aufgrund einer Rechtsnorm des öffentlichen Rechts ergebenden Beziehungen einer Person zu einer anderen Person oder zu einer Sache, auf Grund derer eine der Beteiligten etwas tun muss, kann, darf oder nicht zu tun braucht. ${ }^{18}$

Vorliegend begehrt R die Feststellung, dass der Gemeinderat nicht dazu berechtigt war, ihn auszuschließen. Es steht hier folglich das Nichtbestehen eines (Innen-) Rechtsverhältnisses i.S.d § 43 I VwGO (konkret: der Befugnis zum Ausschluss) in Frage.

\section{Klagebefugnis}

$\mathrm{Zu}$ prüfen ist sodann, ob R gem. § $42 \mathrm{II} \mathrm{VwGO}$ analog auch klagebefugt sein müsste. Eine analoge Anwendung von $\S 42$ II VwGO wird im Rahmen der Feststellungsklage nach $\S 43$ VwGO vielfach für erforderlich gehalten. ${ }^{19}$ In der Sache spricht hierfür, dass das Kommunalverfassungsstreitverfahren ansonsten auf ein objektives Beanstandungsverfahren hinausliefe, das mit dem auf den Schutz subjektiv-

17 Vgl. Becker/Heckmann/Kempen/Manssen/Becker Öffentliches Recht in Bayern, 6. Aufl., Teil 2 Rn. 275.

18 BVerwGE 100, 262 (264f.).

19 BVerwG NVwZ 1989, 470 (470); BVerwG NVwZ 1985, 112 (113); OVG Koblenz BeckRS 2009, 32626; Schoch/Schneider/Bier/Pietzcker VwGO, 32. EL, § 43 Rn. 29; Posser/Wolff/Möstl Beck'scher OnlineKommentar VwGO, 41. Edition, $\S 43$ Rn. 28; a.A. Kopp/Schenke/ Schenke W.-R./Schenke, R. P. VwGO, 22. Aufl., § 42 Rn. 63, die unter Hinweis auf die Eingrenzung des feststellungsfähigen Rechtsverhältnisses das Vorliegen einer planwidrigen Regelungslücke verneinen; s. auch Gärditz/Glaser VwGO-Kommentar, 2013, § 43 Rn. 84 ff.,unter Rekurs auf die bewusste Ausgestaltung der Feststellungsklage als Interessentenklage. öffentlicher Rechte angelegten System der VwGO nicht im Einklang stünde.

Der Meinungsstreit kann freilich dahinstehen, wenn eine Klagebefugnis nach $\S 42$ II VwGO analog ohnehin gegeben wäre. ${ }^{20}$ Dazu müsste $\mathrm{R}$ die Verletzung eines Organrechts ${ }^{21}$ geltend machen. Ausreichend ist insoweit, dass eine Verletzung nicht nach jeder Betrachtung ausgeschlossen ist (Möglichkeitstheorie). Hier erscheint eine Verletzung der Mitwirkungsrechte des R in Form von Beratung und Abstimmung gem. Art. 48 I BayGO möglich. ${ }^{22}$ Diese sollen gerade der Wahrung der Funktion als Bürgervertreter dienen. Als problematisch könnte sich jedoch erweisen, dass bei den Organrechten von staatlichen Kompetenzträgern nicht privates, sondern öffentliches Interesse in Rede steht. ${ }^{23} \mathrm{Zu}$ fordern ist daher eine sogenannte »wehrfähige Innenrechtsposition«. Zu deren Identifizierung ist von G. Kisker die »Kontrastorgantheorie» entwickelt worden. Danach ist erforderlich, dass der Kläger gegenüber dem Beklagten ein partikulares - von sonstigen, auf gleicher Stufe verfolgten Interessen deutlich abzugrenzendes - Sachinteresse verfolgt und dadurch $\mathrm{zu}$ einem sogenannten »Kontrastorgan « im Sinne einer inneradministrativen Gewaltenteilung und -balancierung wird. ${ }^{24}$ Rechtsdurchsetzungsmacht und folglich ein organschaftliches Recht besteht auf Grund solcher Normen, welche die Rechtsposition eines Kontrastorgans konstatieren. ${ }^{25}$ Vorliegend stellt das Teilnahmerecht des $\mathrm{R}$ ein solches organschaftliches Recht dar. ${ }^{26}$

\section{Feststellungsinteresse}

Gemäß § 43 I VwGO muss R ein berechtigtes Interesse an der baldigen Feststellung haben. Als solches kommt jedes als schutzwürdig anerkannte Interesse rechtlicher, wirt-

20 Detterbeck Allgemeines Verwaltungsrecht, 14. Aufl., Rn. 1403. 21 Vgl. hierzu allgemein Becker/Heckmann/Kempen/Manssen/Becker Öffentliches Recht in Bayern, 6. Aufl., Teil 2 Rn. 277.

22 Vgl. Prandl/Zimmermann/Büchner/Pahlke Kommunalrecht in Bayern, Kommentar, 130. EL, Art. 48 GO, S. 2; Lange Kommunalrecht, 2013, Kap 10 Rn. 24.

23 Vgl. Kopp/Schenke/Schenke, W.-R./Schenke, R. P. VwGO, 22. Aufl., $\S 42$ Rn. 80; Schoch/Schneider/Bier/Wahl/Schütz, VwGO, 32. EL, § 42 II Rn. $91 \mathrm{ff}$.

24 Näher Burgi Kommunalrecht, 5. Aufl., §14 Rn. 6; Ogorek JuS 2009, 511 (514); Wysk/Wysk VwGO, 2. Aufl., § 42 Rn. 167; grundlegend Kisker Insichprozeß und Einheit der Verwaltung, 1968, S. 38 ff.; ablehnend dagegen: Ogorek JuS 2009, 511 (514) m.w. N.

25 Schoch/Schneider/Bier/Wahl/Schütz VwGO, 32. EL, § 42 II Rn. 96. 26 Vgl. Schoch/Schneider/Bier/Wahl/Schütz VwGO, 32. EL, § 42 II Rn. 99. 
schaftlicher oder ideeller Art in Betracht. ${ }^{27}$ Da der Ausschluss in der Vergangenheit liegt, müsste dieser, um ein berechtigtes Interesse bejahen zu können, noch in der Gegenwart Wirkung entfalten. Vorliegend könnte eine konkrete Wiederholungsgefahr ${ }^{28}$ in Betracht kommen. Diese liegt vor, wenn konkrete Anhaltpunkte dafür gegeben sind, dass in Zukunft in ähnlicher Weise verfahren wird. ${ }^{29}$ $\mathrm{R}$ wird hier von der Beratung und Entscheidung zum einen hinsichtlich des Zulassungsantrages gegen das Urteil des VG Würzburg und zum anderen über den Erlass der Änderungssatzung ausgeschlossen. Es besteht die Gefahr, dass er auch in Zukunft von Beratungen und Beschlussfassungen aus ähnlichen Gründen ausgeschlossen wird. Konkret ist dies insbesondere für den Fall denkbar, dass die Gemeinde D im Anschluss an ein etwaiges Berufungsurteil über die Einlegung einer Revision erneut Beschluss fassen könnte. Zum anderen kommt ein Rehabilitationsinteresse des R daran in Betracht, den Vorwurf der Befangenheit gerichtlich klären zu lassen. Zwar kann es ein Rehabilitationsinteresse im eigentlichen Sinne im Innenrecht nicht geben, dennoch ist der Gedanke übertragbar. ${ }^{30}$ Denn auf Grund des Öffentlichkeitsgrundsatzes von Gemeinderatssitzungen gem. Art. 52 BayGO erscheint nicht nur eine Verletzung der Organrechte des R möglich, sondern auch eine Herabsetzung seiner Persönlichkeit gegenüber an der Sitzung teilnehmenden Gemeindebürgern. ${ }^{31}$

\section{Beteiligten- und Prozessfähigkeit}

\section{Kläger}

Der Kläger R (gem. § 63 Nr. 1 VwGO) könnte als natürliche Person gem. § 61 Nr. 1 Alt. 1 VwGO beteiligtenfähig sein. Jedoch sind vorliegend nicht die Individualrechte des R, sondern seine innerorganisatorischen Rechte als Organteil, nämlich als Gemeinderatsmitglied betroffen. § $61 \mathrm{Nr} .1$ VwGO ist demnach nicht anwendbar. ${ }^{32}$ Da auch $\S 61 \mathrm{Nr} .3$ VwGO ausscheidet, kommt nur $\S 61 \mathrm{Nr} .2$ VwGO in Betracht. Dieser ist nach richtiger Ansicht für einzelne Gemeinderatsmitglieder analog heranzuziehen. Durch die

27 BVerGE 100, 262 (271); Kopp/Schenke/Schenke, W.-R. VwGO, 22. Aufl., § 43 Rn. 23; Schmidt Kommunalrecht, 2. Aufl., § 15 Rn. 535.

28 Zur Anwendbarkeit der Fallgruppen der Wiederholungsgefahr und des Rehabilitationsinteresses siehe Kopp/Schenke/Schenke, $W .-R$ VwGO, 22. Aufl., § 43 Rn. 23/25.

29 Sinngemäß Detterbeck Allgemeines Verwaltungsrecht, 14. Aufl., Rn. 1426.

30 Ehlers NVwZ 1990, 105 (112).

31 Vgl. VGH Mannheim NVwZ-RR 1997, 181 (182).

$32 \mathrm{Vgl}$. Kopp/Schenke/Schenke, W.-R VwGO, 22. Aufl., § 61 Rn. 5.
Herleitung der Beteiligtenfähigkeit aus der Fähigkeit zur Inhaberschaft des geltend gemachten Rechts drückt die Norm genau das aus, worauf es beim Kommunalverfassungsstreit ankommt. Der Analogieschluss trägt dem Umstand Rechnung, dass - wie eine systematische Gesamtschau aller drei Nummern des $\S 61$ VwGO zeigt - mit »Recht« i.S.d. § 61 Nr. 2 VwGO allein das Außenrecht gemeint ist. ${ }^{33}$ Zudem setzt der Begriff "Vereinigung " (bei direkter Anwendung) sprachlich eine Verbindung von mindestens zwei Gliedern voraus. ${ }^{34} \mathrm{R}$ ist folglich gem. § 61 Nr. 2 VwGO analog beteiligtenfähig.

Die Prozessfähigkeit des R ergibt sich konsequenterweise aus § 62 III VwGO analog, die Vertretung des Organteils erfolgt somit durch ihn als Organwalter. ${ }^{35}$

\section{Beklagter}

$\mathrm{Zu}$ prüfen ist weiterhin, wer Beklagter i.S.d. $\S 63 \mathrm{Nr} .2$ VwGO ist. ${ }^{36}$ Nach dem Rechtsträgerprinzip des $§ 78$ I Nr. 1 VwGO müsste dies die Gemeinde D als Rechtsträgerin des handelnden Gemeinderates sein. Die Gemeinde ist nach $\S 61$ Nr. 1 Alt. 2 VwGO beteiligten- und gemäß $\S 62$ III VwGO prozessfähig. Ihre Vertretung erfolgt durch den ersten Bürgermeister, Art. 38 I BayGO. Fraglich ist jedoch, ob das auf Außenrechtsstreitigkeiten zugeschnittene Rechtsträgerprinzip hier überhaupt Geltung beansprucht. Nach einer in der Literatur vertretenen Ansicht soll bei Innenrechtsstreitigkeiten der vorliegenden Art Beklagter das handelnde Organ selbst sein. ${ }^{37}$ Dies ist hier der Gemeinderat. Dieser wäre gem. § $61 \mathrm{Nr} .2$ VwGO (direkt bzw. analog) beteiligten- und gem. § 62 III VwGO (direkt bzw. analog ${ }^{38}$ ) prozessfähig. Für diese Sichtweise wird vorgebracht, dass

33 Schoch/Schneider/Bier/Bier VwGO, 32. EL, § 61 Rn. 7 m.w.N.; a. A. Ogorek JuS 2009, 511 (516), der eine Beschränkung auf den Außenrechtskreis verneint, i. E. mangels einer »Vereinigung « aber auch eine Analogie annimmt; a. A. OVG Münster NVwZ 1983, 485 (486), welches $\S 61 \mathrm{Nr}$. 2 VwGO direkt anwendet; wieder anders Franz JURA 2005, 156 (160), der $\S 61$ Nr. 1 VwGO analog heranzieht.

34 In diese Richtung auch Burgi Kommunalrecht, 5. Aufl., § 14 Rn. 12.

35 Vgl. Erbguth/Mann/Schubert Besonderes Verwaltungsrecht, 12. Aufl., Rn. 187.

36 Ebenso gut vertretbar ist es, wenn die Frage des richtigen Beklagten erst im Rahmen der Passivlegitimation aufgegriffen wird. Siehe zur Einordnung der Problematik auch Hufen Verwaltungsprozessrecht, 10. Aufl., § 21 Rn. 8.

37 Statt vieler Burgi Kommunalrecht, 5. Aufl., § 14 Rn. 12; Erbguth/ Mann/Schubert Besonderes Verwaltungsrecht, 12. Aufl., Rn. 188.

38 Instruktiv Ogorek JuS 2009, 511 (516), mit Hinweis darauf, dass der Begriff "Vereinigung" nach seinem Wortsinn einen Zusammenschluss zuvor getrennter Individuen voraussetzt. 
der Kommunalverfassungsstreit die Rechtsverhältnisse innerhalb einer Gemeinde betrifft und die zur Rechtsdurchsetzung erforderlichen Handlungen von einem bestimmten Organ vorzunehmen sind. ${ }^{39}$ Nach der überzeugenden Gegenansicht des BayVGH widerspricht ein solcher Ansatz jedoch dem Grundsatz des Rechtsträgerprinzips..$^{40}$ Dies gilt umso mehr, als eine gesetzliche Ausnahme hiervon nicht erkennbar ist. Richtige Beklagte ist daher die Gemeinde als Rechtsträgerin des Gemeinderates.

\section{Allgemeines Rechtsschutzbedürfnis}

Klärungsbedürftig ist sodann im Rahmen des allgemeinen Rechtsschutzbedürfnisses, ob für $\mathrm{R}$ ein einfacherer Weg zur Rechtsverfolgung in Betracht kommt. Ein Beanstandungsverfahren gem. Art. 59 II BayGO scheidet hier aus, da dies nur für den Bürgermeister statthaft ist. Ebenso ist ein Kommunalaufsichtsverfahren gem. Art. 108ff. BayGO vorliegend ohne Bedeutung, weil Einzelne auf den Erlass aufsichtlicher Maßnahmen keinen Anspruch haben. ${ }^{41}$

\section{Zuständigkeit des Gerichts}

Das VG Würzburg ist gem. § $45 \mathrm{VwGO}$ sachlich und gem. $\S 52$ Nr. 5 VwGO i.V.m. Art. 1 II Nr. 5 AGVwGO örtlich zuständig.

\section{B. Begründetheit}

Die Feststellungsklage ist begründet, wenn sie sich gegen den richtigen Beklagten richtet und soweit das streitige Rechtsverhältnis nicht besteht, § 43 I Var. 2 VwGO. Dies ist der Fall, wenn der Beschluss über den Ausschluss des $\mathrm{R}$ rechtswidrig war und dieser dadurch in seinen organschaftlichen Rechten verletzt wurde.

39 Becker/Heckmann/Kempen/Manssen/Becker Öffentliches Recht in Bayern, 6. Aufl., Teil 2 Rn. 279.

40 BayVGH BayVBl. 1984, 77; BayVBl. 1985, 339; BayVBl. 1995, 661 (662).

41 Becker/Heckmann/Kempen/Manssen/Becker Öffentliches Recht in Bayern, 6. Aufl., Teil 2 Rn. 278; Knemeyer Bayerisches Kommunalrecht, 12. Aufl., S. 209 Rn. 288.

\section{Passivlegitimation ${ }^{42}$}

Einigkeit besteht darüber, dass $\S 78$ VwGO mangels Statthaftigkeit einer Anfechtungs- und Verpflichtungsklage keine direkte Anwendung finden kann. ${ }^{43}$ Nach einer Ansicht ist daher auf die allgemeinen Grundsätze für die Prozessführungsbefugnis zurückzugreifen. ${ }^{44} \mathrm{Im}$ Regelfall bedeutet dies, dass das Rechtsträgerprinzip heranzuziehen ist. ${ }^{45}$ Nach überzeugenderer Ansicht ist $\S 78 \mathrm{VwGO}$ analog anzuwenden, da das ihm zu Grunde liegende Rechtsträgerprinzip auch bei anderen Klagebegehren außerhalb von Anfechtungs- und Verpflichtungsklage gilt. ${ }^{46}$ Passivlegitimiert ist folglich gem. § $78 \mathrm{I} \mathrm{Nr} 1 \mathrm{VwGO}$ analog als Rechtsträger des handelnden Gemeinderates die Gemeinde D. ${ }^{47}$

\section{Rechtmäßigkeit des Ausschlusses}

Fraglich ist, ob der Ausschluss rechtmäßig war. Als Ausschlussgründe kommen sowohl Art. 53 I 3 BayGO als auch Art. 49 BayGO in Betracht.

\section{Ausschluss von der Sitzung, Art. 53 I 3 BayGo}

Für den Ausschluss nach Art. 53 I 3 BayGO bedürfte es einer fortgesetzten erheblichen Störung. Eine solche ist aber vorliegend nicht erkennbar.

\section{Persönliche Beteiligung, Art. 49 BayGo}

\section{a) Ermächtigungsgrundlage}

Der Ausschluss könnte aber auf Grundlage des Art. 49 III, I BayGO ${ }^{48}$ erfolgt sein.

42 Zur Einordnung des § 78 VwGO als Element der Begründetheitsprüfung oder als Sachentscheidungsvoraussetzung vgl. Rozek JuS 2007, 601.

43 Engels/Krausnick Kommunalrecht, 2015, Teil 2§ 6 Rn. 23.

44 Kopp/Schenke/Schenke, W.-R VwGO, 22. Aufl., § 78 Rn. 2.

45 Wysk/Saurenhaus/Buchheister VwGO, 2. Aufl., § 78 Rn. 2.

46 Hufen Verwaltungsprozessrecht, 10. Aufl., § 12 Rn. 31.

47 Vgl. zur Diskussion um den richtigen Klagegegner auch bereits die Nachweise in Fn. 37.

48 Parallelvorschriften zB: § 25 III, I HGO; § 18 IV, I GemO BW; § 54 III i.V.m. § 41 I, IV NKomVG; für den Normerlass gilt in Nds. dagegen kein Mitwirkungs-, sondern nur ein Transparenzgebot, § 41 III Nr. 1 i.V.m. IV 3 NKomVG (siehe dazu Schoch/Röhl Besonderes Verwaltungsrecht, 15. Aufl., 1. Kap Rn. 93 mit Fn. 263); § 43 II i. V.m. 31 IV, I GO NRW; § 20 III, I SächsGemO. 


\section{b) Formelle Rechtmäßigkeit}

Die Zuständigkeit des Gemeinderates ergibt sich aus Art. 49 III BayGO. ${ }^{49}$ Verfahrens- oder Formfehler sind nicht ersichtlich.

\section{c) Materielle Rechtmäßigkeit}

Die materielle Rechtmäßigkeit des Ausschlusses setzt voraus, dass R oder eine in Art. 49 I BayGO genannte Person sowohl aus der Entscheidung über den Berufungszulassungsantrag als auch aus dem Erlass der Änderungssatzung einen unmittelbaren Vor- oder Nachteil i.S. d. Art. 49 I BayGO ziehen könnte. Hier kommt jeweils nur eine Betroffenheit des R selbst in Betracht, so dass die persönliche Voraussetzung zu bejahen ist. ${ }^{50}$ Des Weiteren müsste für ihn die Möglichkeit eines unmittelbaren (bb) und individuellen (cc) Vor- oder Nachteils (aa) bestehen. ${ }^{51}$

\section{aa) Konkrete Möglichkeit eines Vor- oder Nachteils}

Zunächst müsste das Bestehen eines Vor- oder Nachteils für R in Betracht kommen. Da Art. 49 BayGO neben dem Schutz des betreffenden Gemeinderatsmitgliedes vor einem Interessenkonflikt das Ziel verfolgt, schon den bösen Anschein eigennützigen Verhaltens in der Kommunalverwaltung zu verhindern, ${ }^{52}$ reicht die konkrete Möglichkeit der persönlichen Betroffenheit bereits aus. Hierauf deutet im Übrigen auch der Wortlaut der Norm (»bringen kann«) hin. Damit wird zugleich dem Umstand Rechnung getragen, dass zu Beginn der Beratung über einen Tagesordnungspunkt das Ergebnis nicht absehbar ist und auch nach der Beschlussfassung offen sein kann, ob der Voroder Nachteil tatsächlich eintreten wird..$^{53}$

49 § 25 III HGO; § 18 IV 2 GemO BW, § 54 IV i. V.m. § 41 IV 2 NKomVG (Vertretung); § 43 II Nr. 4 i.V.m. § 31 IV 2 GoNRW; § 20 III 2 SächsGemO.

50 Parallelvorschriften in § 25 I Nr. 1 HGO; § 18 I GemO BW; § 54 III i.V.m. § 41 I Nr. 1 NKomVG; § 43 II i.V.m. 31 I Nr. 1 GO NRW; § 20 I SächsGemO.

51 Näher zu den Voraussetzungen eines Ausschlusses wegen persönlicher Beteiligung: Becker/Heckmann/Kempen/Manssen/Becker Öffentliches Recht in Bayern, 6. Aufl., Teil 2 Rn. 247 ff.; die Erläuterung der »individuellen Betroffenheit" kann auch im Rahmen des Tatbestandsmerkmals des Vor- oder Nachteils, vgl. dazu Prandl/Zimmermann/Büchner/Pahlke Kommunalrecht in Bayern, Kommentar, 130. EL, Art. 49 GO, S. 5, bzw. der Unmittelbarkeit erfolgen, vgl. dazu Kingreen JURA (JK) 2015, 315; Hölzl/Hien/Huber GO mit VGemO, LKrO und BezO für den Freistaat Bayern, 57. EL, Art. 49 GO, S. 8.

52 Prandl/Zimmermann/Büchner/Pahlke Kommunalrecht in Bayern, Kommentar, 130. EL, Art. 49 GO, S. 1; Röhl JURA 2006, 725 (730).

$53 \mathrm{Hölzl} /$ Hien/Huber GO mit VGemO, LKrO und BezO für den Freistaat Bayern, 57. EL, Art. 49 GO, S. 8.

\section{(1) Berufungszulassungsantrag}

Fraglich ist zunächst, ob $\mathrm{R}$ durch den Beschluss über die Einlegung der Berufung einen Vor- oder Nachteil materieller, persönlicher oder ideeller Art erlangen kann. ${ }^{54}$

Die Entscheidung des Gemeinderates über die Rechtsmitteleinlegung gegen das im Musterverfahren ergangene Urteil müsste zunächst R selbst betreffen. Für den Fall, dass der Gemeinderat auf die Einlegung eines Rechtsmittels verzichtet, wäre das Urteil rechtskräftig geworden. Es würde somit zugleich präjudizierende - und zwar begünstigende - Wirkung für die ruhenden Klageverfahren der übrigen 58 Kläger entfalten. $R$ kann deshalb gerade nicht einwenden, es gehe bei der Entscheidung des Gemeinderates formal nicht um »sein« Verfahren, sondern ausschließlich um das Vorgehen der Gemeinde im Hinblick auf das Musterverfahren. Denn ein solches Pilotverfahren soll grundsätzliche Rechtsfragen klären, um aus dem Prozessergebnis für die übrigen, zum Ruhen gebrachten Verfahren Konsequenzen ziehen zu können. ${ }^{55}$

Der Verzicht auf die Einlegung eines Rechtsmittels wäre folglich für $\mathrm{R}$ vorteilhaft. Ein Berufungsverfahren könnte für den R nachteilige Auswirkungen haben.

\section{(2) Änderungssatzung}

Klärungsbedürftig ist im Weiteren, ob auch die Verabschiedung der Änderungssatzung für R vor- oder nachteilig sein könnte. Mit dem Erlass der Satzung sollten die angefochtenen Bescheide rückwirkend auf eine neue Rechtsgrundlage gestellt und die beanstandeten Mängel geheilt werden. Auf diese Weise würde sichergestellt, dass die Kläger zu den Wasser-, Abwasser- und Abfallgebühren in den streitigen Jahren herangezogen werden können. Das Musterverfahren hätte in einer zweiten Instanz keinen Erfolg. Zugleich würde ein positiver Ausgang der übrigen 58 anhängigen Klagen verhindert. ${ }^{56}$ Der Erlass könnte somit für R nachteilig sein.

\section{bb) Unmittelbarkeit}

Der mögliche Vor- oder Nachteil müsste sich des Weiteren als unmittelbare Folge des Beschlusses darstellen. Hiervon ist auszugehen, wenn er sich aus diesem selbst, seinem Vollzug oder einem von ihm eingeleiteten Verfahren er-

54 Vgl. allgemein Prandl/Zimmermann/Büchner/Pahlke Kommunalrecht in Bayern, Kommentar, 130. EL, Art. 49 GO, S. 5; Becker/Heckmann/Kempen/Manssen/Becker Öffentliches Recht in Bayern, 6. Aufl., Teil 2 Rn. 248.

55 VGH Kassel NVwZ-RR 2014, 563 (564). 56 VGH Kassel NVwZ-RR 2014, 563 (565). 
gibt. ${ }^{57}$ Es ist mithin nicht erforderlich, dass der Gemeinderatsbeschluss i.S. einer direkten Kausalität ohne weiteren Zwischenschritt den möglichen Vor- oder Nachteil herbeiführt. Ein derart enges Verständnis würde dazu führen, dass Art. 49 I 1 BayGO weitgehend leer liefe, da Beschlüsse des Gemeinderates gem. Art. 36 S. 1 BayGO in der Regel des Vollzugs bedürfen..$^{58}$ Im Lichte des Normzwecks der Vermeidung schon des bösen Anscheins eigennützigen Verhaltens genügt es vielmehr, dass der Beschluss ein Verfahren in Gang setzt, an dessen Ende der Eintritt des Voroder Nachteils steht. Maßgeblich sollte eine adäquate Kausalität sein..$^{59}$ Eine Bedingung ist adäquat kausal, wenn sie nach der Lebenserfahrung unter den jeweiligen Umständen des Einzelfalls geeignet ist, den Vor- oder Nachteil herbeizuführen. ${ }^{60}$

\section{(1) Berufungszulassungsantrag}

Der mögliche Vor- oder Nachteil müsste unmittelbar auf Grund des Beschlusses über den Berufungszulassungsantrag eintreten. Eine direkte Kausalität könnte nur bejaht werden, wenn der Gemeinderat beschließt, auf eine Berufung zu verzichten. Dies wäre für $\mathrm{R}$ unmittelbar vorteilhaft. Wenn hingegen die Entscheidung getroffen wird, dass eine Berufung eingelegt wird, wäre eine direkte Kausalität zu verneinen, da dies für $\mathrm{R}$ keine konkreten Auswirkungen hätte. Erst der tatsächliche Antrag der Berufung und das folgende Verfahren könnten, je nach Ausgang, für R positive oder negative Wirkung mit sich bringen.

Da eine direkte Kausalität, wie bereits erläutert, jedoch mit Blick auf Sinn und Zweck des Art. 49 I 1 BayGO kein tragfähiges Kriterium darstellt, zumal selbst offensichtliche Fälle der Verfolgung eigener Interessen gegebenenfalls nicht vom Mitwirkungsverbot umfasst wür-

57 BayVGH VerwRspr 1960, 478 (482).

58 Prandl/Zimmermann/Büchner/Pahlke Kommunalrecht in Bayern, Kommentar, 130. EL, Art. 49 GO, S. 5; Hölzl/Hien/Huber GO mit VGemO, LKrO und BezO für den Freistaat Bayern, 57. EL, Art. 49 GO, S. 9; der niedersächsische Gesetzgeber hat mit §54 III i.V.m. § 41 I 2 NKomVG eine Definition der Unmittelbarkeit normiert und darin ausdrücklich festgehalten, dass die bloße Ausführung von Beschlüssen keine Maßnahme ist, die das Unmittelbarkeitskriterium entfallen lässt.

59 Lissack Bayerisches Kommunalrecht, 3. Aufl., S. 178 Rn. 59.

60 Prandl/Zimmermann/Büchner/Pahlke Kommunalrecht in Bayern, Kommentar, 130. EL, Art. 49 GO, S. 5; VGH Kassel NVwZ-RR 2014, 563 (564); a.A. noch VGH Kassel NVwZ 1982, 44 (45), wo eine direkte Kausalität gefordert wird; zur Änderung der Rspr des hessischen VGH siehe ausführlicher Lange LKRZ 2014, 221 (221f.); gut vertretbar erscheint die Forderung nach einer direkten Kausalität insb. in NRW, wo das Kriterium der Unmittelbarheit in § 31 I 2 GO NRW bewusst enggefasst wird. den, ${ }^{61}$ ist zu prüfen, ob eine adäquate Kausalität bejaht werdenkann. Es ist somit keine formale, sondern eine materiell wertende Sicht einzunehmen. ${ }^{62}$ Gegen diese Betrachtungsweise kann auch nicht vorgebracht werden, dass nur eine formale Sicht den Gemeindeorganen die notwendige Rechtssicherheitbei Entscheidungenüber die Teilnahmean Beschlussfassungen biete. Denn auch an anderen Stellen in der GO wird den Gemeinderäten der Umgang mit unbestimmten Rechtbegriffen abverlangt. ${ }^{63} \mathrm{Da}$ der Gemeinderat durch die Entscheidung ohne weiteres ermächtigt wird, erforderliche Rechtsschritte $\mathrm{zu}$ unternehmen, ${ }^{64}$ ist der Beschluss geeignet, einen Vor- oder Nachteil herbeizuführen, so dass dieadäquate Kausalität zu bejahen ist.

Das Kriterium der Unmittelbarkeit ist somit im Hinblick auf den Berufungszulassungsantrag erfüllt.

\section{(2) Änderungssatzung}

Zwar ist ein möglicher Nachteil auf Grund des Beschlusses über den Satzungserlass nicht ohne weitere Zwischenschritte denkbar, da erst ein Heranziehungsbescheid zu einer Beitragspflicht führen würde. ${ }^{65}$ Eine direkte Kausalität müsste folglich bei rein formaler Betrachtung verneint werden. Da aber - wie gezeigt - gerade nicht allein darauf abzustellen ist, ob die Entscheidung eine Person ohne Hinzutreten weiterer Umstände direkt berührt, ${ }^{6}{ }^{6}$ könnte die Unmittelbarkeit vorliegend dennoch anzunehmen sein. Auf Grundlage der Satzung können Gebührenbescheide ergehen. Die Satzung wäre somit adäquat kausal und die Unmittelbarkeit ist zu bejahen. ${ }^{67}$

\section{cc) Individuelle Betroffenheit ${ }^{68}$}

Der Vor- oder Nachteil muss zudem individueller Natur sein. Dafür reicht ein Gruppenvorteil oder -nachteil nicht aus. Vielmehr muss ein Sondervorteil oder -nachteil beste-

61 VGH Kassel NVwZ-RR 2014, 563 (564).

62 Waldhoff JuS 2014, 1150 (1151f.).

63 Siehe dazu zB: Hölzl/Hien/Huber GO mit VGemO, LKrO und BezO für den Freistaat Bayern, 57. EL, Art. 37 GO, S. 3; BayObLG BayObLGZ 1974, 374 (376f.).

64 VGH Kassel BeckRS 2014, 46680.

65 VGH Kassel BeckRS 2014, 46680.

66 So auch schon: VGH Kassel NVwZ-RR 2014, 563 (564).

67 Dazu auch: Prandl/Zimmermann/Büchner/Pahlke Kommunalrecht in Bayern, Kommentar, 130. EL, Art. 49 GO, S. 5.

68 Im Gegensatz zu Art. 49 I 1 BayGO wird in § 25 I 2 HGO, § 18 III GemO BW, § 54 III i. V.m. § 41 I 3 NKomVG, § 43 II i. V.m. 31 III Nr. 1 GO NRW, § 20 II Nr. 2 SächsGemO die Wahrnehmung eines Gruppeninteresses als Ausnahme vom Mitwirkungsverbot ausdrücklich normiert. 
hen. Entscheidend ist also, ob jemand als Einzelner oder nur als Teil einer Gruppe betroffen ist. ${ }^{69}$ Je allgemeiner und abstrakter sich die zur Eingrenzung der Personenmehrheit dienenden Merkmale gestalten und je größer die Anzahl der Interessenträger ist, desto eher ist ein bloßer Gruppenvorteil anzunehmen. ${ }^{70}$

\section{(1) Berufungszulassungsantrag}

Vorliegend würde die Entscheidung über den Berufungsantrag nicht nur den $\mathrm{R}$, sondern auch die anderen 58 Kläger betreffen. Wirkt sich, wie hier, die Maßnahme für eine Personenmehrheit aus, muss die Möglichkeit bestehen, dass das Gemeinderatsmitglied einen individuellen Sondervorteil aus der Sache ziehen könnte, der nur ihm alleine zu Gute kommt und gerade der Grund für die Zweifel an einer ordnungsgemäßen Kommunalverwaltung ist. Nicht ausreichend ist es hingegen, wenn jemand als Angehöriger einer Berufs- oder Bevölkerungsgruppe beteiligt ist, deren gemeinsame Interessen durch die Angelegenheiten berührt werden. ${ }^{71}$ Als Bevölkerungsgruppe ist eine größere Anzahl von Gemeindeeinwohnern anzusehen, die ein gemeinsames, d.h. im Wesentlichen identisches, Interesse verbindet. ${ }^{72}$ Dies ist bei den 59 Klägern fraglich. Jeder der Kläger ist gegen den Gebührenbescheid gerichtlich vorgegangen. Klärungsbedürftig ist aber, ob dadurch nicht dennoch nur ein Kollektivinteresse gegeben ist, welches für einen Ausschlussgrund nicht ausreichen würde. Die Annahme einer Gruppe scheidet - wobei die Grenzen fließend verlaufen - jedoch aus, wenn die Folgen einer Entscheidung von vornherein individualisierbar sind. Dies ist $\mathrm{zu}$ bejahen, sofern sich eine Entscheidung derart auf den kommunalen Mandatsträger zuspitzt, dass er als Adressat der Entscheidung angesehen werden kann. $\mathrm{R}$ ist zwar »nur« einer von 59 Klägern, aber jeder Kläger versucht die für ihn und sein Grundstück für die Vergangenheit festgesetzte Gebührenlast zu vermindern, weswegen es überzeugend erscheint, ein gemeinsames (politisches) Interesse $\mathrm{zu}$ verneinen (a.A. vertretbar). Wenn es sich lediglich um ein Bündel von Individualinteressen handelt, die jeder

69 Prandl/Zimmermann/Büchner/Pahlke Kommunalrecht in Bayern, Kommentar, 130. EL, Art. 49 GO, S. 5/6; Becker/Heckmann/Kempen/ Manssen/Becker Öffentliches Recht in Bayern, 6. Aufl., Teil 2 Rn. 250. 70 Prandl/Zimmermann/Büchner/Pahlke Kommunalrecht in Bayern, Kommentar, 130. EL, Art. 49 GO, S. 6.

71 VGH Kassel NVwZ-RR 2014, 563 (565); Hölzl/Hien/Huber GO mit VGemO, LKrO und BezO für den Freistaat Bayern, 57. EL, Art. 49 GO, S. 10.

72 VGH Kassel NVwZ-RR 2014, 563 (565). im eigenen Interesse verfolgt, ist $R$ nicht als Vertreter einer Bevölkerungsgruppe, sondern individuell betroffen. ${ }^{73}$

\section{(2) Änderungssatzung}

Beim Beschluss von Satzungen kann in der Regel kein Sondervorteil entstehen, ${ }^{74}$ da der Erlass abstrakt-genereller Regelungen den Gemeindevertreter grundsätzlich nicht personenbezogen trifft. Vielmehr belasten Abgabensatzungen all diejenigen gleichermaßen, die einen abgaberechtlichen Tatbestand der Satzung erfüllen. ${ }^{75}$ Fraglich ist, ob dies hier anders beurteilt werden kann. Wendet sich die Satzung nur an ganz wenige Betroffene, lässt sich auch dann von einem Individualinteresse sprechen, wenn die Maßnahme äußerlich eine Gruppe anspricht. ${ }^{76}$ Vorliegend könnte eine Sonderbetroffenheit gegenüber den übrigen Gemeindebürgern, die zur Zahlung von Abwasser- und Abfallgebühren allgemein verpflichtet sind, dadurch entstanden sein, dass der Kläger die ergangenen Abgabenbescheide angefochten hat. Der Gemeinderat wollte mit dem Erlass der Satzungen verhindern, dass diejenigen, die gegen die Bescheide vorgegangen sind, auf Grund eines obsiegenden Urteils nicht mehr zur Zahlung der Beiträge herangezogen werden können. Es sollte damit also im Fall eines für die Kläger positiven Ausgangs der Verfahren, eine Grundlage geschaffen werden, dennoch Gebühren einholen zu können. Da die Bescheide der übrigen Einwohner bereits bestandskräftig geworden waren, hätte die Satzung keine Auswirkungen über den Kreis der Klagenden hinaus und beträfe somit nur eine begrenzte Anzahl individuell Betroffener. Bei einer Unterscheidung rückwirkender oder zukünftiger Änderungen der Gebührensatzungen wird deutlich, dass erstere gerade nur die 59 Fälle betreffen. ${ }^{77}$ Zwar misst der hier zugrunde gelegte Ansatz dem Gedanken der unparteiischen Amtsführung ein höheres Gewicht bei, als dem "parlamentsrechtlichen « Grundsatz der freien Mandatsausübung. Beide Grundsätze stehen beim Erlass von Satzungen in einem Spannungsverhältnis. Auf der einen Seite ist mit Blick auf die Funktionsfähigkeit der gemeindlichen Selbstverwaltungsorgane ein gewisses persönliches

73 VGH Kassel NVwZ-RR 2014, 563 (565).

$74 \mathrm{Hölzl} / \mathrm{Hien} /$ Huber GO mit VGemO, LKrO und BezO für den Freistaat Bayern, 57. EL, Art. 49 GO, S. 10; Becker/Heckmann/Kempen/ Manssen/Becker, Öffentliches Recht in Bayern, 6. Aufl., Teil 2 Rn. 251. 75 VGH Kassel NVwZ-RR 2014, 563 (565).

$76 \mathrm{Hölzl} /$ Hien/Huber GO mit VGemO, LKrO und BezO für den Freistaat Bayern, 57. EL, Art. 49 GO, S. 10; Prandl/Zimmermann/Büchner/ Pahlke Kommunalrecht in Bayern, Kommentar, 130. EL, Art. 49 GO, S. 6. 77 VGH Kassel NVwZ-RR 2014, 563 (566). 
Engagement der Gemeinderatsmitglieder hinzunehmen, da ein abstrakt-genereller Beschluss des Gemeinderats naturgemäß auch deren Mitglieder betreffen kann. Auf der anderen Seite ist der Gemeinderat kein Parlament im staatsorganisatorischen Sinne, sondern das "Selbstverwaltungsorgan der Exekutive «, ${ }^{78}$ weshalb einer mandatsfreundlichen Auslegung nicht zwangsläufig der Vorrang einzuräumen ist. Dies bedeutet vorliegend auch nicht, dass es dem $\mathrm{R}$ verwehrt wird, privat gegen die Bescheide vorzugehen und sich zugleich politisch für eine Satzungsänderung $\mathrm{zu}$ engagieren. Denn $\mathrm{R}$ ist nicht daran gehindert sich für zukünftige Änderungen der Gebührensatzung einzusetzen. Die rückwirkende Änderung betrifft aber gerade nur die 59 Kläger, denn dadurch wurde ein individualisierbarer, in der Vergangenheit abgeschlossener Vorgang neu geregelt. ${ }^{79}$ Vorliegend kann deshalb erneut eine individuelle Betroffenheit des $\mathrm{R}$ bejaht werden.
Nach hier vertretener Auffassung handelt es sich um ein individuelles Sonderinteresse, so dass die individuelle Betroffenheit zu bejahen ist.

\section{dd) Zwischenergebnis}

Der Gemeinderat war berechtigt, den R von der Gemeinderatssitzung auf Grund des Art. 49 III, I BayGO auszuschließen. Der Beschluss war demnach materiell rechtmäßig.

\section{Verletzung organschaftlicher Rechte}

Im Lichte der materiellen Rechtmäßigkeit des Beschlusses konnte $\mathrm{R}$ durch diesen auch nicht in seinen organschaftlichen Rechten verletzt werden.

\section{Ergebnis}

Da zwar die Sachentscheidungsvoraussetzungen vorliegen, die Klage aber unbegründet ist, hat diese keine Aussicht auf Erfolg.
78 VGH Kassel NVwZ-RR 2014, 563 (566).

79 VGH Kassel NVwZ-RR 2014, 563 (566). 